
3 Research Square
Preprints are preliminary reports that have not undergone peer review.
They should not be considered conclusive, used to inform clinical practice, or referenced by the media as validated information.

\title{
TaLBD1, a LOB Transcription Factor Gene In $T$. Aestivum, Improves Plant N Starvation Adaptation Via Modulating N Acquisition-Associated Processes
}

\section{Yanyang Zhang}

Hebei Agricultural University

\section{Chenyang Ni}

Hebei Agricultural University

Tianjiao Li

Hebei Agricultural University

Le Han

Hebei Agricultural University

Pingping Du

Hebei Agricultural University

Kai Xiao ( $\nabla$ xiaokai@hebau.edu.cn )

Agricultural University of Hebei https://orcid.org/0000-0002-3295-9860

\section{Research Article}

Keywords: LOB type transcription factor, N starvation, Transgene analysis, N uptake, Root system architecture establishment

Posted Date: December 28th, 2021

DOI: https://doi.org/10.21203/rs.3.rs-1118467/v1

License: (c) (1) This work is licensed under a Creative Commons Attribution 4.0 International License. Read Full License 


\section{Abstract}

Members of transcription factor (TF) families contribute largely to plant $\mathrm{N}$ starvation tolerance by regulating downstream stress defensive genes. In this study, we characterized TaLBD1, a Lateral Organ Boundary (LOB) TF gene in T. aestivum, in regulating plant low- $\mathrm{N}$ stress adaptation. TaLBD1 harbors the conserved domains specified by plant LOB proteins, targeting onto nucleus after endoplasmic reticulum (ER) assortment. The TaLBD1 transcripts were response sensitively to $\mathrm{N}$ starvation (NS) signaling, showing to be gradually upregulated in aerial and root tissues over a 27-h NS condition. The N. tabacum lines overexpressing TaLBD1 improved phenotype, root system architecture (RSA) establishment, biomass, and N contents of plants under NS treatment. The nitrate transporter gene NtNRT2.4 and PINFORMED gene NtPIN6 significantly upregulated in expression in NS-challenged lines; knockdown expression of NtNRT2.4 decreased N uptake and that of NtPIN6 alleviated RSA establishment relative to WT. These results validate the function of NRT and PIN genes in regulating plant N uptake and RSA behavior. RNA-seq analyses revealed that a quantity of genes modify expression in N-deprived lines overexpressing $T a L B D 1$, which enriched into functional groups of signal transduction, transcription, protein biosynthesis, primary or secondary metabolism, and stress defensiveness. These findings suggested that the TaLBD1-improved NS adaptation attributes largely to its role in transcriptionally regulating NRT and PIN genes as well as in modulating those functional in various biological processes. $T a L B D 1$ is a crucial regulator in plant $\mathrm{N}$ starvation tolerance and valuable target for molecular breeding high $\mathrm{N}$ use efficiency (NUE) crop cultivars.

\section{Key Message}

\section{The LOB TF member TaLBD1 in T. aestivum confers plants $\mathbf{N}$ starvation tolerance via modulation of $\mathbf{N}$ uptake and RSA establishment.}

\section{Introduction}

Nitrogen $(\mathrm{N})$ acts as of one the indispensible inorganic nutrients for all of the living organisms. The conditions of $\mathrm{N}$ supply impact largely on growth, development, and productivity potential of the plants. In several of past decades, application of $\mathrm{N}$ fertilizers contributed greatly to the sustainable crop production development around the world; however, lowered N use efficiency (NUE) in crop plants is accompanied with the overdosed $\mathrm{N}$ fertilizers, which failed utilization efficiently due to the nature of $\mathrm{N}$ nutrition being prone to be leaching and evaporation, leading to intensified pollution of environment aside from the elevated production investment. Therefore, improving the NUE for crop plants has been an essential issue for promotion of the sustainable agriculture worldwide (Wang et al. 2019; Laurens et al. 2021).

The acquisition of $\mathrm{N}$ and the internal translocation of it across various tissues in plants are accomplished underlying mediation of diverse physiological and biochemical processes. Under the $\mathrm{N}$ starvation (NS) condition, a large set of genes functional in various categories including those encoding $\mathrm{N}$ signaling receptors, kinases, transcription factors (TF), and stress-defensive proteins, display modified efficiencies 
on transcription or translation (Kant et al. 2011; Lang et al. 2014; Antal et al. 2016; Wang et al. 2019). These components operate synergistically in mediating the plant NS response through modulating the NS defensiveness-associated biological processes.

Acted as one of the large families of TF, the members of Lateral Organ Boundary (LOB) Domain (LBD) family are plant specific that have been evolved from the same progenitor with charophyte algae (Coudert et al. 2012; Chanderbali et al. 2015). The proteins of the LOB family are specified by a LOB domain (also designated as AS2) consisting of following conserved domains: a C-motif, a Gly-Ala-Ser (GAS) block, and a leucine-zipper-like coiled-coil motif (Ohashi et al. 2018). Among these, the C-motif functions in binding the cis-acting regulatory elements situated in the downstream gene promoters whereas the coiled-coil motif involves the interaction processes between LBD TF and other types of proteins. Previously, extensive investigations have suggested that the LOB members play essential roles in regulating various physiological processes associated with growth, development, and abiotic stress responses of plants, including leaf primordium differentiation (Ohashi-lto et al. 2018; Zhang et al. 2020), cellular secondary metabolism (Zhang et al. 2020), lateral root formation (Okushima et al. 2007), microspore cell division (Yang et al. 2021), and N signaling transduction once plant perception of the modifying environmental $\mathrm{N}$ availability (Kimura et al. 2017). These results together suggest that the LOB TF members function as essential modulators in mediating various biological processes in plants.

The property of $\mathrm{N}$ uptake and characterization of root system architecture (RSA) are two determinants in plant adaptation to $\mathrm{N}$ starvation (NS) conditions (Yusefi-Tanha et al. 2020). Thus far, a subset of nitrate transporter (NRT) members that contribute to plant tolerance to low-N stress has been reported (YusefiTanha et al. 2020). In addition, the RSA feature has been documented to be regulated synergistically by a set of external factors, including the localization, concentration and translocation of phytohormone auxin at cellular level (Brunetti et al. 2018; Doyle et al. 2019). To this issue, distinct members of PIN-FORMED (PIN) family were recorded to be involved in polar transportation of the cellular auxin, playing critical roles in modulation of physiological processes associated with initiation and elongation of primary and lateral roots, by which to contribute to plant NS tolerance (Ye et al. 2001; Baetsen et al. 2021). However, the molecular processes as to how the NRT members regulate $\mathrm{N}$ uptake and weather the PIN proteins mediate RSA acclimation to low-N stress underlying LOB TF modulation are needed to be further characterized.

Wheat ( $T$. aestivum) is one of the important cereal crops to provide huge food source for humanity around the world. Todate, although the LBD TF family members have been identified and subjected to functional characterization in some plant species, such as $A$. thaliana and $O$. sativa (Evans et al. 2007; Soyano et al. 2008; Matsumura et al. 2009), the members of this TF family in T. aestivum remain largely to be investigated. In this study, we characterized TaLBD1, a gene of the LOB TF family in wheat, in mediating plant adaptation to NS stress. Our findings suggest that $T a L B D 1$ sensitively responds to external $\mathrm{N}$ starvation condition at transcriptional level and confers improved plant NS tolerance by improving $\mathrm{N}$ uptake and RSA establishment of plants challenged with low-N stress. 


\section{Materials And Methods}

\section{Characterization analysis on TaLBD1}

Our previous RNA-seq analyses aimed at elucidating profiles of transcriptome in $T$. aestivum (cv. Shinong 086) upon low-N stress identified TaLBD1, a member of the LBB transcription factor family (GenBank accession No. AK330221), displayed significantly upregulated transcription (unpublished data). This finding encouraged us to further investigate its molecular characterization given the potential function of TF in mediating stress response in plants. The homologous genes of TaLBD1 distributed in various plant species were obtained based on BLASTn search against with the GenBank database in NCBI (https://www.ncbi.nlm.nih.gov). The phylogenetic relations among TaLBD1 and its plant counterparts were established using the MegAlign algorithm supplemented in the DNAStar software.

\section{Subcellular location analysis of the TaLBD1 protein}

The subcellular localization of TaLBD1 after endoplasmic reticulum (ER) assortment was predicted using an online tool referred to as NL Stradamus. Moreover, additional experiment was performed based on transgene analysis to validate the prediction results of the subcellular localization of target protein. With this purpose, the open reading frame (ORF) of TaLBD1 was amplified using RT-PCR together with gene specific primers (Table S1), which was then integrated in upstream the ORF of reporter gene (GFP) in

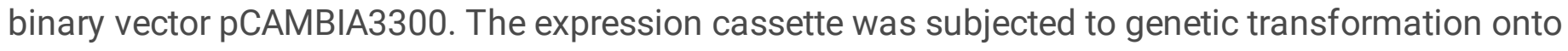
epidermal cells of $N$. tabacum (cv. Wisconsin 35) using the A. Tumefaciens-mediated approach as described by (Keen et al. 2020). The GFP signals initiated from fusion TaLBD1-GFP across whole cell were detected under fluorescent microscope by which to define the location of target protein at subcelluar level.

\section{Expression analysis of TaLBD1}

The roots and leaves of $T$. aestivum (cv. Shinong 086) treated with varied $\mathrm{N}$ levels were subjected to evaluation of TaLBD1 transcripts. To this end, wheat seedlings were cultured in a standard Murashige and Skoog (MS) solution (affluent N, $16 \mathrm{mM}$ ) as previously described by (Jiang et al. 2006). At the thirdleaf growth stage, the NS treatment was set up for the seedlings by culturing them in a modified MS solution supplemented with lowered $\mathrm{N}$ supply (NS, $0.06 \mathrm{mM} \mathrm{N}$ ). Tissues of roots and leaves were sampled at $0 \mathrm{~h}$ (prior to treatment), and $1 \mathrm{~h}, 3 \mathrm{~h}, 9 \mathrm{~h}$, and $27 \mathrm{~h}$ after the NS treatment. In addition, an $\mathrm{N}$ recovery treatment was established to address the target gene response to recovered normal $\mathrm{N}$ condition. For that, an aliquot of the seedlings after $27 \mathrm{~h}$ of NS were re-cultured in a standard MS solution. The roots and leaves were collected after $1 \mathrm{~h}, 3 \mathrm{~h}, 9 \mathrm{~h}$, and $27 \mathrm{~h}$ during the $\mathrm{N}$ recovery condition. The TaLBD1 transcripts in collected tissues were evaluated based on qPCR performed to be similarly to previously described by Guo et al. (2013), using gene specific primers (Table S1). Tatubulin, a constitutive gene in T. aestivum, was used as an internal reference to normalize the target transcripts (Table S1). 
TaLBD1 was overexpressed in an ectopic species (i.e., N. tabacum) to define its function in mediating plant NS response. With this purpose, the ORF of TaLBD1 was amplified using RT-PCR together with the gene specific primers (Table S1). It was then inserted into the restriction sites BgAl/ BstEl in vector pCAMBIA3301 under the control of the CaMV35S promoter. The procedure for generating transgenic lines was similar to that reported previously (Sun et al. 2012).

Line 2 and Line 3, two lines at T3 generation with high expression level of TaLBD1, were selected to define the gene function in regulating NS response of the plants. With this purpose, the transgenic lines and wild type (WT) plants were subjected to two $\mathrm{N}$ level treatments, including affluent $\mathrm{N}$ (AN) by culturing in a standard MS solution (16 mM N) and NS treatment by growing in a modified MS solution supplemented with lowered $\mathrm{N}(0.3 \mathrm{mM} \mathrm{N})$. The growth conditions for plants in two $\mathrm{N}$ level treatments were as follows: a photoperiod of $14 \mathrm{~h} / 10 \mathrm{~h}$ (light/dark) with light intensity of $400 \mu \mathrm{E} / \mathrm{m}^{2} \mathrm{~s}$ during light phase, temperature of $26^{\circ} \mathrm{C} / 22^{\circ} \mathrm{C}$ (light/dark), and relative air humidity from $60 \%$ to $75 \%$. During culture process, the solutions were air-circulated using a mini pump and renewed twice for each week. Six weeks after treatments, the plant growth traits of transgenic lines and WT, including phenotype, biomass, fresh weights and volumes of roots were assessed. Among these, phenotypes of plants and root tissues were recorded based on a digital camera; biomass of plants and root tissues was obtained from three representative plants after conventional oven-drying; fresh weights and root volumes of root tissues were determined according to the conventional approach. In addition, a set of photosynthetic parameters, including photosynthetic rate (Pn), photosystem II photochemical efficiency (UPSII), and nonphotochemical quenching coefficient (NPQ), were measured in the transgenic lines and WT plant after N level treatments. Of which, $\mathrm{Pn}, \mathrm{gs}$, and $\mathrm{Ci}$ were measured using the photosynthesis system (LiCOR-6200) following the manufacturer's suggestion; $\Psi P S I l$ and NPQ were assessed as reported previously (Guo et al. 2013).

\section{Assay of the $\mathrm{N}$ contents and expression patterns of the NRT family genes}

The $\mathrm{N}$ contents and the expression patterns of a suite of nitrate transporter (NRT) family genes involving $\mathrm{N}$ uptake were analyzed in transgenic lines after $\mathrm{N}$ level treatments, by which to address the gene function in mediating plant $\mathrm{N}$ nutrition under NS. Of which, $\mathrm{N}$ concentrations were measured as described by Guo et al. (2013). Accumulative $\mathrm{N}$ amounts in plants were calculated by multiplying the $\mathrm{N}$ concentrations and plant biomass. The NRT family genes in N. tabacum that were subjected to the expression analysis included NtNRT1.1-s, NtNRT1.1-t, NtNRT2.5, and NtNRT2.6. Transcripts of the NRT family genes in transgenic and WT plants were evaluated using qRT-PCR together with corresponding gene-specific primers (Table S1). Nttubulin, a constitutive gene in N. tabacum, was used as an internal reference to normalize the target transcripts.

\section{Assay of the expression patterns of the PIN-FORMED family genes}

Cellular auxin level controls largely on the root system architecture (RSA) establishment of plants, playing an important role in mediating water and nutrient acquisition of root tissues under abiotic stress 
conditions (Brunetti et al. 2018; Doyle et al. 2019). Members of the PIN-FORMED family act as the critical mediator in regulating internal auxin translocation and the cellular auxin level, by which to affect RSA establishment (Gray et al. 2001; Reed et al. 2001). To understand the putative PIN family members that contributed to modified RSA feature underlying TaLBD1 regulation, we indentified the genes of PINFORMED family genes in N. tabacum, namely, NtPIN1, NtPIN1b, NtPIN6, and NtPIN9, and evaluated expression levels of them in the transgenic lines under NS treatment. To this end, qRT-PCR was performed to assess the transcripts of these PIN family genes. Gene specific primers used for amplification of them are listed in Table S1. Nttubulin was used as internal reference to normalize the target transcripts.

\section{Transgene analysis on distinct NRT and PIN-FORMED family genes}

The NRT family gene NtNRT2.4 and the PIN-FORMED family gene NtPIN6 displayed significantly upregulated expression in N-deprived transgenic lines (i.e., Line2 and 3), suggesting their putative involvement in mediating plant $\mathrm{N}$ uptake and RSA establishment. Therefore, we performed transgene analysis on them to characterize their functions in mediating $\mathrm{N}$ uptake and RSA establishment, respectively. With this purpose, the ORF in anti-sense orientation of NtNRT2.4 and NtPIN6 were separately amplified based on RT-PCR using gene specific primers (Table S1). They were then inserted into restriction sites $\mathrm{Ncol} / \mathrm{BstEl}$ in vector pCAMBIA3301 under the control of the CaMV35S promoter as aforementioned. The lines with significant knockdown expression of target genes were established to be similarly for generating the TaLBD1 overexpression lines. Three transgenic lines designated as NtNRT2.41, NtNRT2.4-3 and NtNRT2.4-4 for NtNRT2.4 and two lines AnPIN6-1 and AnPIN6-2 for NtPIN6, were selected and subjected to two $\mathrm{N}$ level treatments as mentioned above (i.e., AN with $16 \mathrm{mM} \mathrm{N}$ and NS with $0.3 \mathrm{mM} \mathrm{N}$ ). Six weeks after treatments, the phenotypes, biomass, $\mathrm{N}$ concentrations and $\mathrm{N}$ accumulative amounts in NtNRT2.4 lines were assessed. Likewise, the phenotypes of plants and root tissues, plant biomass, and fresh weights and volumes of root tissues were evaluated in NtPIN6 lines. The Nassociated traits and root growth traits were assessed to be similarly to those performed in the TaLBD1 overexpression lines mentioned above.

\section{Transcriptome analysis}

High-throughput RNA-seq analyses were performed to characterize the transcriptiome profile underlying modulation of TaLBD1 under the NS condition. To this end, Line 2, the transgenic line overexpressing TaLBD1 together with WT were cultured regularly in a standard MS solution as aforementioned. At the fifth leaf stage, they were subjected to NS treatment for another one week. Total RNA in Line 2 and WT plants was extracted using TRIzol reagent (Invitrogen) and subjected to construction of RNA-seq libraries following the procedure as described previously (Zhong et al. 2011). Transcripts generated in the RNA-seq libraries were sequenced using the Illumina HiSeq 2500 system. Valuable transcripts in libraries generated from the N-deprived transgenic lines and WT were obtained by removing the adaptors in reads, the reads with sequence length less than $40 \mathrm{bp}$, and those being low quality based on the software Trimmomatic (Bolger et al. 2014). These clean reads were then subjected to alignment analysis against the database for transcripts of the reference genome ( $N$. tabacum, Novogene Co, LTd, Beijing). We defined 
the genes to be differentially expressed (DE) when they exhibited 2-fold variation on transcripts across the transgenic and WT plants (Robinson et al. 2010), using a false discovery rate (FDR) less than 0.05 (Benjamini and Hochberg 1995). The DE genes were categorized into distinct GO terms using the online tool referred to as Plant MetGenMap (http://bioinfo.bti.cornell.edu/cgi-bin/MetGenMAP/home.cgi), in which a CPAN pearl module was applied as described previously (Boyle et al. 2004). Functional groups of the $\mathrm{DE}$ genes identified in transgenic lines were determined based on gene $\mathrm{GO}$ annotations.

\section{Expression analysis on randomly selected DE genes in RNAseq analysis}

Ten of DE genes identified in the RNA-seq analyses, including five to be upregulated and five downregulated, were subjected to evaluation of transcripts based on qPCR using gene specific primers (Table S1), to validate the RNA-seq analysis results. The five genes with upregulated expression pattern included those encoding mitogen-activated protein kinase kinase, leucine zipper protein, ribosomal protein L3A, malate dehydrogenase, and peroxidase; the five ones with downregulated expression pattern were those coding for cytokinin-regulated kinase, WARK protein, phosphoglyceromutase, metal transporter, and chitinase. cDNA samples derived from Line 2 and WT after NS treatment were used as the templates in qRT-PCR. Likewise, the constitutive Nttubulin was used as an internal reference to normalize target transcripts.

\section{Statistical analysis}

Averages of plant and root biomass, $\mathrm{N}$ concentration, $\mathrm{N}$ accumulative amount, root fresh weight, root volume and the expression levels were all derived from triplicate results. Standard errors of averages and significant differences among the averages were analyzed using the Statistical Analysis System software.

\section{Results}

\section{The characterization of TaLBD1}

The full length cDNA of TaLBD1 is 1267 bp that encodes a 303-aa polypeptide (Fig. S1). Similar to the members of LOB TF family in plant species, the TaLBD1 protein harbors a conserved C-motif (CX2CX6CX3C) (5 aa-19 aa) at $\mathrm{N}$ terminus (Fig. S2). Based on phylogenetic relation analysis, it was revealed that $T a L B D 1$ shares high similarities to the homologous genes distributed in diverse plant species at nucleic acid level, including those from H.vulgare, P.edulis, O. sativa, Z. mays, I. triloba, B. nivea and $A$. thaliana (with identities changing from 76.5 to $95.8 \%$, Fig. 1). TaLBD1 targets onto nucleus after endoplasmic reticulum (ER) assortment based on online prediction analysis, which is consistent with result of the experiment indicating that the GFP signals derived from fusion TaLBD1-GFP were confined in nucleus of $N$. tabacum epidermal cells (Fig. 2A). Subcellular localization of the TaLBD1 protein onto nucleus is in agreement with its TF nature exerting roles in regulating transcription of the downstream genes. 


\section{TaLBD1 expression is response sensitively to the NS treatment}

The expression levels of TaLBD1 were drastically altered in both roots and leaves once challenged with NS condition. Under affluent $\mathrm{N}$ condition (AN), the transcripts of TaLBD1 in tissues examined were low. In contrast, they were increased dramatically under NS treatment, being gradually elevated over a $27 \mathrm{~h} \mathrm{NS}$ regime treatment and reaching a peak at end of the treatment (Fig. 2B). Moreover, the upregulated transcripts of TaLBD1 upon NS were restored steadily in tissues following a $27 \mathrm{~h}$ of $\mathrm{N}$ recovery treatment (Fig. 2B). These results together suggested that TaLBD1 is sensitive in response to Nsignaling condition and possibly involves the NS signaling transduction in plants.

\section{TaLBD1 confers plants significantly improved tolerance to the low-N stress}

Two tobacco lines at T3 generation, namely, Line 2 and Line 3 with strong expression of TaLBD1 (Fig. S3), were subjected to two treatments with contrasting $N$ levels. Under AF condition, comparable growth traits, including phenotypes and biomass in plants and roots were observed in both the transgenic lines and WT (Figs. 3A). Under NS treatment, however, the transgenic lines significantly improved phenotype (Fig. 3A), RSA establishment (Fig. 3B), and biomass in both plants and roots (Fig. 3C) compared with WT. In line with above growth traits, the transgenic lines displayed improved photosynthetic parameters, such as elevated photosynthetic rate (Pn), enhanced photosystem II biochemical efficiency (UPSII), and reduced nonphotochemical quenching (NPQ) with respect to WT (Figs. 3D-3F). These results suggested that $T a L B D 1$ acts as a crucial regulator in enhancing plant tolerance to the low-N stress.

\section{Overexpression of TaLBD1 confers plant improved $\mathrm{N}$ accumulation capacity}

$\mathrm{N}$-associated traits in the transgenic lines (i.e., Lines 2 and 3) and WT were assessed after the $\mathrm{N}$ level treatments. Under AN condition, similar to the growth traits mentioned above, the transgenic lines were comparable on $\mathrm{N}$ concentrations and $\mathrm{N}$ accumulative amounts with WT (Figs. 4A-4B). Under NS treatment, all of the transgenic lines (Lines 2 and 3 ) and WT were lower on $\mathrm{N}$ concentrations and the $\mathrm{N}$ accumulative amounts than shown under the $\mathrm{N}$ normal condition (Figs. 4A-4B). However, Lines 2 and 3 increased $\mathrm{N}$ concentrations and $\mathrm{N}$ accumulative amounts compared to WT under NS treatment (Figs. 4A4B). Behaviors of these $\mathrm{N}$-associated traits in transgenic lines are consistent with the growth traits and photosynthetic parameters shown under contrasting $\mathrm{N}$ level treatments, suggesting that the TaLBD1improved plant $\mathrm{N}$ tolerance is largely attributed to the gene function in positively regulating $\mathrm{N}$ uptake of plants challenged with low- $\mathrm{N}$ stress.

\section{Expression patterns of NRT genes and their function in mediating plant NS adaptation}

Expression patterns of a suite of NRT family genes were analyzed in the N-deprived transgenic and WT plants to understand the molecular processes underlying $\mathrm{N}$ uptake mediated by TaLBD1. Among nine genes of NRT genes in N. tabacum examined, NtNRT2.4 was significantly upregulated in expression in the transgenic lines with respect to WT (Fig. 5A). Its modified expression pattern upon N starvation was in contrast to other NRT genes whose transcripts were similar in both transgenic and WT plants (Fig. 5A). 
Therefore, NtNRT2.4 is a gene to be putatively regulated underlying TaLBD1 regulation at transcription level and contributes to the NS adaptation of the N-deprived transgenic lines.

Three typical lines with NtNRT2.4 knockdown expression (NRT2.4-1, NtNRT2.4-3 and NRT2.4-4) (Fig. S4) were subjected to characterization of gene function in regulating $\mathrm{N}$ uptake. Under NS treatment, these lines alleviated significantly on phenotypes (Fig. 5B), biomass, $\mathrm{N}$ concentrations, and accumulated $\mathrm{N}$ amounts in plants compared with WT (Figs. 5C-5E). These results suggested that NtNRT2.4 functions effectively in mediating the $\mathrm{N}$ uptake by impacting on $\mathrm{N}$ accumulation and growth-associated processes of the plants treated by low-N stress.

\section{Expression patterns of PIN-FORMED family genes and their roles in mediating root RSA establishment}

Transcripts of ten genes in PIN-FORMED family in N. tabacum, were evaluated in the N-deprived transgenic lines (Lines 2 and 3) and WT to understand weather any of them involves RSA establishment underlying TaLBD1 regulation. In contrast to other PIN genes whose transcripts were similar each other in transgenic lines and WT plants, NtPIN6 displayed significantly upregulated expression in Lines 2 and 3 relative to WT (Fig. 6A). These results suggested that NtPIN6 is transcriptionally regulated by TaLBD1 and contributes to the improved RSA establishment in transgenic lines challenged with NS condition.

Transgene analysis on NtPIN6 was performed to characterize its function in regulating RSA establishment. Under NS treatment, NtPIN6-1 and NtPIN6-2, two typical lines with NtPIN6 knockdown expression (Fig. S5) significantly alleviated root growth (Fig. 6B), reduced biomass, decreased root fresh weights, and lowered root volumes of the plants compared to WT plants (Figs. 6C-6E). These results together suggested that distinct genes in PIN-FORMER family, such as NtPIN6, positively impact on RSA establishment in plants overexpressing TaLBD1 treated by NS condition.

\section{The differentially expressed (DE) genes underlying regulation of TaLBD1}

The DE genes in lines overexpressing TaLBD1 were identified under $\mathrm{N}$ starvation condition based on a high-throughput RNA-seq analysis. A total of 1971 genes were differentially expressed in transgenic line (i.e., Line 2) under NS treatment with respect to WT. Among these, 962 were categorized into the expression pattern of upregulated and 1009 of downregulated (Fig. 7A, Dataset 1-Dataset 2). To verify the results derived from the transcriptome datasets, we analyzed the transcripts of ten DEGs randomly selected from the datasets, including five upregulated and another five downregulated shown in the Ndeprived transgenic lines. All of the DE genes displayed expression levels with comparable variation-folds shown in the transcriptome analyses. These results validated the nature of reproducibility in our transcriptome analysis (Fig. 8). Therefore, TaLBD1 acts as an important regulator in plant $\mathrm{N}$ starvation signaling transduction system which modulates transcription of downstream genes at global level.

Among the DE genes identified in transcriptome analyses, only small ratio of them has been annotated to known biological functions thus far (including a total of 130 upregulated and 161 downregulated) (Dataset 1-Dataset 2). That the DEGs with a large ratio identified to be function unknown is suggested 
due to the limitation of the gene annotation programs in $N$. tabacum species. Based on the annotated biological functions, the upregulated DEGs are categorized into following functional groups: signal transduction (with numbers of 16), transcriptional regulation (15), protein metabolism (14), chromosome remodeling (5), primary metabolism (26), secondary metabolism (11), transport (2), oxidative stress defense (4), phytohormone response (6), abiotic stress response (7), biotic stress response (4), cellular structure (10), cell cycling (4), development (3), and miscellaneous (3) (Fig. 7B; Dataset 3). Likewise, the downregulated DEGs with annotated functions are classified into following functional groups: signaling transduction (20), transcriptional regulation (8), protein metabolism (9), chromosome remodeling (6), primary metabolism (29), secondary metabolism (19), transport (7), phytohormone response (6), abiotic stress response (5), biotic stress response (12), cellular structure (14), cell cycling (2), development (2), and miscellaneous (22) (Fig. 7C; Dataset 4). The results on transcriptome analyses in N-deprived transgenic plants suggested that $T a L B D 1$ exerts roles in regulating transcription of numerous genes, which are involved in various biological functions by synergistically acting in physiological processes associated with plant $\mathrm{N}$ starvation tolerance.

\section{Discussion}

The members of the LOB transcription factor family play critical roles in mediating physiological processes associated with plant growth, development, and stress responses, involving modulation of leaf polarity establishment (Zhu et al. 2020; Busche et al. 2021), tracheary element development (Soyano et al. 2008), boundary delimitation (Ohashi et al. 2015; Ohashi et al. 2018), cytokinin signaling, inflorescence branch formation (Guo et al. 2020), female gametophyte development (Evans et al. 2007), KNOX gene regulation (Semiarti et al. 2001; Chalfun et al. 2005; Long et al. 2014; Long et al. 2015), and N deprivation adaptation (Rubin et al. 2009). In this study, our characterization on TaLBD1, a gene of the LOB TF family in T. aestivum, revealed its high similarities at CDNA level with the homologous genes across diverse plant species. The conserved domains situated in TaLBD1 and the subcellular localization onto nucleus of the protein are consistent with members of the LOB TF family, which exert putative biological functions in nucleus through regulation of the downstream genes at transcriptional level.

Plant response to NS is closely associated with transcriptional modulation of the $\mathrm{N}$ deprivationresponsive genes. Previously, a set of cis-acting regulatory elements such as nitrate-responsive elements (NRE), was identified to be situated in promoters of the $\mathrm{N}$ uptake- and assimilation-associated genes, contributing to modified transcription efficiency of NS-responsive genes (Jian et al. 2018). For instance, NRT2.1 and NRT2.2, two genes of NRT family in A. thaliana, displayed induction on expression upon low$\mathrm{N}$ stress, which is closely associated with interaction of the NRE motifs in them with distinct TFs (Jian et al. 2018). In this study, our expression analysis on TaLBD1 indicated that its transcripts are response to low-N stress in aerial and root tissues, which suggests its involvement in mediating NS response of the plants. Further characterization on cis-acting regulatory elements situated in the promoter of this $\angle O B$ gene can deepen understanding the transcriptional mechanism of it upon NS condition in T. aestivum. 
The function of $\angle O B$ members in mediating transduction of $\mathrm{N}$ deprivation signaling has been recorded (Rubin et al. 2009). In this study, we selected N. tabacum as an ectopic expression system to generate transgenic lines with $T a L B D 1$ overexpression, by which to characterize putative role of target gene in mediating plant NS response. Results indicated that the transgenic lines (i.e., Line 2 and Line 3) significantly improved low-N stress tolerance with respect to control, the wild type (WT) without subjected to genetic transformation of target gene. The transgenic lines showed much better on phenotype, biomass of aerial tissues and roots, fresh weight and volume of root tissues under NS treatment compared with WT. These results are in agreement with behavior of the improved photosynthetic parameters, such as elevated $\mathrm{Pn}$, enhanced photosystem II biochemical efficiency, and reduced nonphotochemical quenching (NPQ) in the N-deprived lines overexpressing TaLBD1. Together, our findings suggest that $T a L B D 1$ acts as an essential regulator in mediating the plant $\mathrm{N}$ starvation response..

Plant low-N stress tolerance is largely dependent on the capacity of $\mathrm{N}$ uptake of plants challenged with NS condition. It has been clearly documented that nitrate transporter (NRT) system consisting of members of the high-affinity transport (HAT) exerts crucial role in regulating $\mathrm{N}$ accumulation in $\mathrm{N}$ deprived plants (Remans et al. 2006; Da et al. 2019). Characterization on HAT system has revealed that members of the RT2 group act as critical components, which contribute to the $\mathrm{N}$ acquisition capacity of plants under low-N conditions (Wang et al. 2020). For example, the mutants nrt2.1 with knockout of AtNRT2.1, a gene of NRT2 family in Arabidopsis, reduces HATS activity and decreases $\mathrm{N}$ content relative to wild type (Li et al. 2007). In this study, we assessed the $\mathrm{N}$ accumulation property in transgenic lines (i.e., Lines 2 and 3) under NS treatment to understand the physiological processes associated with TaLBD1-mediated low-N stress tolerance improvement. Higher $\mathrm{N}$ concentration and more accumulative amounts of $\mathrm{N}$ were observed in $\mathrm{N}$-deprived transgenic plants than WT. These findings suggest that TaLBD1 confers plant enhanced $\mathrm{N}$ uptake by which to improve plant NS tolerance. Our expression analysis on NRT family genes in N. tabcucm revealed that NtNRT2.4 enhances expression levels significantly in N-deprived transgenic lines relative to WT. Moreover, using transgenic lines with NtNRT2.4 knockdown expression, we experimentally verifieded the NRT gene function in mediating plant NS response. Compared with WT, the lines with knockdown expression of NtNRT2.4 dramatically alleviated growth traits and lowered $\mathrm{N}$ accumulative amounts of the plants under NS treatment. These results suggest that distinct NRT genes underlying control of TaLBD1 to constitute putative action modules, such as LBD1-NRT2.4, which contribute to plants improved $\mathrm{N}$ uptake and tolerance to low-N stress.

Auxin acts as one of critical members of phytohormones and involves multiple biological processes associated with plant growth and development, including initiation and formation of lateral roots to impact on nutrient and water acquisition of plants (Brunetti et al. 2018). The auxin signaling involving modulation of various physiological processes is transduced through synergistic actions of diverse biochemical pathways (Gray et al. 2001; Doyle et al. 2019). Thus far, it has been documented that members of the PIN-FORMED family play essential roles in controlling the auxin levels and distribution patterns in cells, acting as an important determinant in modulating establishment of RSA (Rogers et al. 2016; Lee et al. 2021). In this study, our characterization on RSA in transgenic lines overexpressing 
TaLBD1 revealed that the target gene confers plants enlarged RSA feature under NS treatment, suggesting that the TaLBD1-mediated plant NS tolerance is also ascribed to its regulation on RSA establishment. In addition, based on expression analysis, the members of PIN-FORMED family with modified transcription in N-deprived lines were identified. In contrast to other genes with unaltered transcripts in transgenic and WT plants, NtPIN6 displayed significantly upregulated expression in Ndeprived lines with TaLBD1 overexpression compared to WT; transgene analysis on this PIN gene validated its function in significantly improving RSA establishment of plants challenged with the NS condition. These findings together suggest that the putative pathway LBD1-PIN6-RSA acts as another mechanism for low-N stress tolerance in plants underlying regulation of TaLBD1. Recently, it has been documented that distinct members of the LOB TF family are involved in auxin signaling transduction pathways, which exert biological roles in mediating formation of lateral root tissues (Filleur et al. 2001). Further investigation on mechanisms as to how distinct PIN-FORMED family members modulate RSA establishment mediated by LOB TF members can provide insights into molecular processes associated with plant root development and low-N stress adaptation.

High throughput transcriptome analysis provides effective approach to elucidate molecular processes underlying the plant stress response (Nemhauser et al. 2004; Lang et al. 2014; Keen et al. 2020). In this study, based on RNA-seq analysis, we investigated the genes with modified transcription in N-deprived transgenic lines overexpressing TaLBD1. As a result, a total of 1971 genes, including 962 to be upregulated and 1009 downregulated, were identified. These differentially expressed (DE) genes are categorized into various functional groups, including signal transduction, transcriptional regulation, protein biosynthesis and degradation, primary and secondary metabolism, stress response, and phytohormone response, etc. These results suggested that TaLBD1 mediates plant low-N stress tolerance to be also associated with its function in modulating the biological processes mentioned above, aside from its regulation on N uptake (i.e., via function module LBD1-NRT2.4) and improvement on RSA establishment (via biochemical pathway LBD1-PIN6-RSA formation). Further characterization on the DE genes playing critical regulatory roles in plant stress response underlying regulation of TaLBD1 can benefit to elucidate the biochemical pathways contributing to plant $\mathrm{N}$ starvation adaptation in crop plants.

\section{Conclusion}

TaLBD1 harbors the conserved domains shared by members of the plant LOB TF family, targeting onto nucleus after endoplasmic reticulum (ER) assortment. TaLBD1 is sensitive in response to low-N stress treatment, showing induced transcripts upon $\mathrm{N}$ starvation in aerial and root tissues and whose induced expression under NS was recovered following $\mathrm{N}$ recovery treatment. TaLBD1 confers plants improved growth traits treated by low-N stress, displaying to be better on RSA, biomass production, photosynthetic function, and N accumulation for plants. Distinct genes in NRT family referred to as NtNRT2.4 and PINFORMED family NtPIN6 significantly upregulated in expression in N-deprived lines overexpressing $T a L B D 1$, exerting roles in the TaLBD1-mediated improvement of plant low-N stress tolerance by enhancing $\mathrm{N}$ uptake and RSA establishment. RNA-seq analysis identified large sets of genes with 
significantly modification on transcription underlying regulation of $T a L B D 1$, which are categorized into functional groups associated with signal transduction, transcription, protein biosynthesis, primary or secondary metabolism, and stress defensiveness, etc. TaLBD1 is a valuable target gene for genetic engineering of high NUE crop cultivars that are cultivated under the N-saving conditions.

\section{Declarations}

\section{Author contribution statement}

Kai Xiao designed the research. Yanyang Zhang, Chenyang Ni, Tianjiao Li, Le Han, and Pingping Du conducted the experiment and performed data analysis. Kai Xiao wrote the paper. All authors contributed to the paper and approved the final manuscript.

\section{Acknowledgements}

This work was supported by the National Natural Science Foundation of China (No. 31872869) and the Science and Technology Planning Project of Hebei Province (No. 216Z6401G).

\section{Compliance with ethical standards}

Conflict of interest The authors declare that they have no conflict of interest.

\section{References}

1. Antal T, Kurkela J, Parikainen M, Kårlund A, Hakkila K, Tyystjärvi E, Tyystjärvi T (2016 Jun) Roles of Group 2 Sigma Factors in Acclimation of the Cyanobacterium Synechocystis sp. PCC 6803 to Nitrogen Deficiency. Plant Cell Physiol 57(6):1309-1318

2. Baetsen-Young A, Chen H, Shiu SH, Day B Contrasting transcriptional responses to Fusarium virguliforme colonization in symptomatic and asymptomatic hosts.Plant Cell. 2021 Apr17;33(2):224-247

3. Brunetti C, Fini A, Sebastiani F, Gori A, Tattini M (2018 Jul) Modulation of Phytohormone Signaling: A Primary Function of Flavonoids in Plant-Environment Interactions. Front Plant Sci 20:9:1042

4. Busche M, Scarpin MR, Hnasko R, Brunkard JO TOR coordinates nucleotide availability with ribosome biogenesis in plants. Plant Cell. 2021 Jul 2;33(5):1615-1632

5. Chalfun-Junior A, Franken J, Mes JJ, Marsch-Martinez N, Pereira A, Angenent GC (2005) ASYMMETRIC LEAVES2-LIKE1 gene, a member of the AS2/LOB family, controls proximal-distal patterning in Arabidopsis petals. Plant Mol Biol 57:559-575

6. Chanderbali AS, He F, Soltis PS, Soltis DE (2015) Out of the water: Origin and diversification of the LBD gene family. Mol Biol Evol 32:1996-2000

7. Coudert Y, Dievart A, Droc G, Gantet P (2012) ASL/LBD phylogeny suggests that genetic mechanisms of root initiation downstream of auxin are distinct in Lycophytes and Euphyllophytes. Mol Biol Evol 
30:569-572

8. Da Cunha AC, Gomes LS, Godoy-Santos F, Faria-Oliveira F, Teixeira JA, Sampaio GMS, Trópia MJM, Castro IM, Lucas C, Brandão RL (2019 May) High-affınity transport, cyanide-resistant respiration, and ethanol production under aerobiosis underlying efficient high glycerol consumption by Wickerhamomyces anomalus. J Ind Microbiol Biotechnol 46(5):709-723

9. Doyle SM, Rigal A, Grones P, Karady M, Barange DK, Majda M, Pař́zková B, Karampelias M, Zwiewka M, Pěnčík A, Almqvist F, Ljung K, Novák O, Robert S (2019) A role for the auxin precursor anthranilic acid in root gravitropism via regulation of PIN-FORMED protein polarity and relocalisation in Arabidopsis. New Phytol. Aug;223(3):1420-1432

10. Evans MMS (2007) The indeterminate gametophyte1 gene of maize encodes a LOB domain protein required for embryo sac and leaf development. Plant Cell 19:46-62

11. Filleur S, Dorbe MF, Cerezo M, Orsel M, Granier F, Gojon A et al (2001) An arabidopsis t-dna mutant affected in nrt2 genes is impaired in nitrate uptake. FEBS Lett 489:0-224

12. Guo T, Lu ZQ, Shan JX, Ye WW, Dong NQ, Lin HX (2020 Sep) ERECTA1 Acts Upstream of the OsMKKK10-OsMKK4-OsMPK6 Cascade to Control Spikelet Number by Regulating Cytokinin Metabolism in Rice. Plant Cell 32(9):2763-2779

13. Jian S, Liao Q, Song H, Liu Q, Lepo JE, Guan C, Zhang J, Ismail AM, Zhang Z (2018 Dec) NRT1.1Related NH4+ Toxicity Is Associated with a Disturbed Balance between NH4+ Uptake and Assimilation. Plant Physiol 178(4):1473-1488

14. Kant S, Bi Y-M, Rothstein SJ (2011) Understanding plant response to nitrogen limitation for the improvement of crop nitrogen use efficiency. J Exp Bot 62:1499-1509

15. Keen P, Hastings AP, Agrawal AA, Van Eck J (2020 Mar) Agrobacterium tumefaciens-Mediated Transformation of Three Milkweed Species (Asclepias hallii, A. syriaca, and A. tuberosa: Apocynaceae). Curr Protoc Plant Biol 5(1):e20105

16. Kimura S, Waszczak C, Hunter K, Wrzaczek M (2017 Apr) Bound by Fate: The Role of Reactive Oxygen Species in Receptor-Like Kinase Signaling. Plant Cell 29(4):638-654. doi: 10.1105/tpc.16.00947. Epub 2017 Apr 3. PMID: 28373519; PMCID: PMC5435433

17. Kuijt SJ, Greco R, Agalou A, Shao J, 't Hoen CC, Overnäs E, Osnato M, Curiale S, Meynard D, van Gulik R, de Faria Maraschin S, Atallah M, de Kam RJ, Lamers GE, Guiderdoni E, Rossini L, Meijer AH, Ouwerkerk PB (2014 Apr) Interaction between the GROWTH-REGULATING FACTOR and KNOTTED1LIKE HOMEOBOX families of transcription factors. Plant Physiol 164(4):1952-1966

18. Lang SI, Aerts R, van Logtestijn RS, Schweikert W, Klahn T, Quested HM, van Hal JR, Cornelissen JH (2014 Jun) Mapping nutrient resorption efficiencies of subarctic cryptogams and seed plants onto the Tree of Life. Ecol Evol 4(11):2217-2227

19. Laurens LML, Olstad JL, Templeton DW (2020) Total Protein Content Determination of Microalgal Biomass by Elemental Nitrogen Analysis and a Dedicated Nitrogen-to-Protein Conversion Factor. Methods Mol Biol. ;1980:233-242. doi: 10.1007/7651_2018_126. PMID: 29675783 
20. Lee H, Ganguly A, Baik S, Cho HT Calcium-dependent protein kinase 29 modulates PIN-FORMED polarity and Arabidopsis development via its own phosphorylation code.Plant Cell. 2021 Nov4;33(11):3513-3531

21. Li W, Wang Y, Okamoto M, Crawford NM, Siddiqi MY, Glass ADM (2007) Dissection of the AtNRT2.1:AtNRT2.2 inducible high-affinity nitrate transporter gene cluster. Plant Physiol 143:425433

22. Long Y, Smet W, Cruz-Ramírez A, Castelijns B, de Jonge W, Mähönen AP, Bouchet BP, Perez GS, Akhmanova A, Scheres B, Blilou I (2015 Apr) Arabidopsis BIRD Zinc Finger Proteins Jointly Stabilize Tissue Boundaries by Confining the Cell Fate Regulator SHORT-ROOT and Contributing to Fate Specification. Plant Cell 27(4):1185-1199

23. Nemhauser JL, Mockler TC, Chory J (2004) Interdependency of brassinosteroid and auxin signaling in Arabidopsis. PLoS Biol 2:1460-1471

24. Ohashi-Ito K, Iwamoto K, Fukuda H, LOB DOMAIN-CONTAINING (2018) PROTEIN 15 Positively Regulates Expression of VND7, a Master Regulator of Tracheary Elements. Plant Cell Physiol. May 1;59(5):989-996

25. Ohashi-Ito K, Iwamoto K, Fukuda H, LOB DOMAIN-CONTAINING (2018) PROTEIN 15 Positively Regulates Expression of VND7, a Master Regulator of Tracheary Elements. Plant Cell Physiol. May 1;59(5):989-996

26. Okushima Y, Fukaki H, Onoda M, Theologis A, Tasaka M (2007) ARF7 and ARF19 regulate lateral root formation via direct activation of LBD/ASL genes in Arabidopsis. Plant Cell 19:118-130

27. Remans T, Nacry P, Pervent M, Girin T, Tillard P, Lepetit M et al (2006) A central role for the nitrate transporter NRT2.1 in the integrated morphological and physiological responses of the root system to nitrogen limitation in Arabidopsis. Plant Physiol 140:909-921

28. Rogers ED, Monaenkova D, Mijar M, Nori A, Goldman DI, Benfey PN (2016 Jul) X-Ray Computed Tomography Reveals the Response of Root System Architecture to Soil Texture. Plant Physiol 171(3):2028-2040

29. Rubin G, Tohge T, Matsuda F, Saito K, Scheible WR (2009) Members of the LBD family of transcription factors repress anthocyanin synthesis and affect additional nitrogen responses in Arabidopsis. Plant Cell 21:3567-3584

30. Semiarti E, Ueno Y, Tsukaya H, Iwakawa H, Machida C, Machida Y (2001) The ASYMMETRIC LEAVES2 gene of Arabidopsis thaliana regulates formation of a symmetric lamina, establishment of venation and repression of meristem-related homeobox genes in leaves. Development 128:17711783

31. Soyano T, Thitamadee S, Machida Y, Chua NH (2008) ASYMMETRIC LEAVES2-LIKE19/ LATERAL ORGAN BOUNDARIES DOMAIN30 and ASL20/ LBD18 regulate tracheary element differentiation in Arabidopsis. Plant Cell 20:3359-3373

32. Wang P, Wang Z, Pan Q, Sun X, Chen H, Chen F, Yuan L, Mi G Increased biomass accumulation in maize grown in mixed nitrogen supply is mediated by auxin synthesis.J Exp Bot. 2019 
Mar27;70(6):1859-1873. doi: 10.1093/jxb/erz047. PMID: 30759246; PMCID: PMC6436159.

33. Wang X, Wang HF, Chen Y, Sun MM, Wang Y, Chen YF (2020 Nov) The Transcription Factor NIGT1.2 Modulates Both Phosphate Uptake and Nitrate Influx during Phosphate Starvation in Arabidopsis and Maize. Plant Cell 32(11):3519-3534

34. Wang Y, Cao JJ, Wang KX, Xia XJ, Shi K, Zhou YH, Yu JQ, Zhou J (2019 Feb) BZR1 Mediates Brassinosteroid-Induced Autophagy and Nitrogen Starvation in Tomato. Plant Physiol 179(2):671685

35. Yang F, Fernández-Jiménez N, Tučková M, Vrána J, Cápal P, Díaz M, Pradillo M, Pecinka A (2021 Sep) Defects in meiotic chromosome segregation lead to unreduced male gametes in Arabidopsis SMC5/6 complex mutants. Plant Cell 24;33((9):3104-3119

36. Ye J, Wang X, Hu T, Zhang F, Wang B, Li C, Yang T, Li H, Lu Y, Giovannoni JJ, Zhang Y, Ye Z (2017 Sep) An InDel in the Promoter of Al-ACTIVATED MALATE TRANSPORTER9 Selected during Tomato Domestication Determines Fruit Malate Contents and Aluminum Tolerance. Plant Cell 29(9):22492268

37. Yusefi-Tanha E, Fallah S, Rostamnejadi A, Pokhrel LR (2020) Root System Architecture, Copper Uptake and Tissue Distribution in Soybean (Glycine max (L.) Merr.) Grown in Copper Oxide Nanoparticle (CuONP)-Amended Soil and Implications for Human Nutrition. Plants (Basel). Oct 8;9(10):1326

38. Zhang Y, Li Z, Ma B, Hou Q, Wan X Phylogeny and Functions of LOB Domain Proteins in Plants.Int J Mol Sci. 2020 Mar26;21(7):2278

39. Zhu F, Deng J, Chen H, Liu P, Zheng L, Ye Q, Li R, Brault M, Wen J, Frugier F, Dong J, Wang T (2020 Sep) A CEP Peptide Receptor-Like Kinase Regulates Auxin Biosynthesis and Ethylene Signaling to Coordinate Root Growth and Symbiotic Nodulation in Medicago truncatula. Plant Cell 32(9):28552877

\section{Figures}




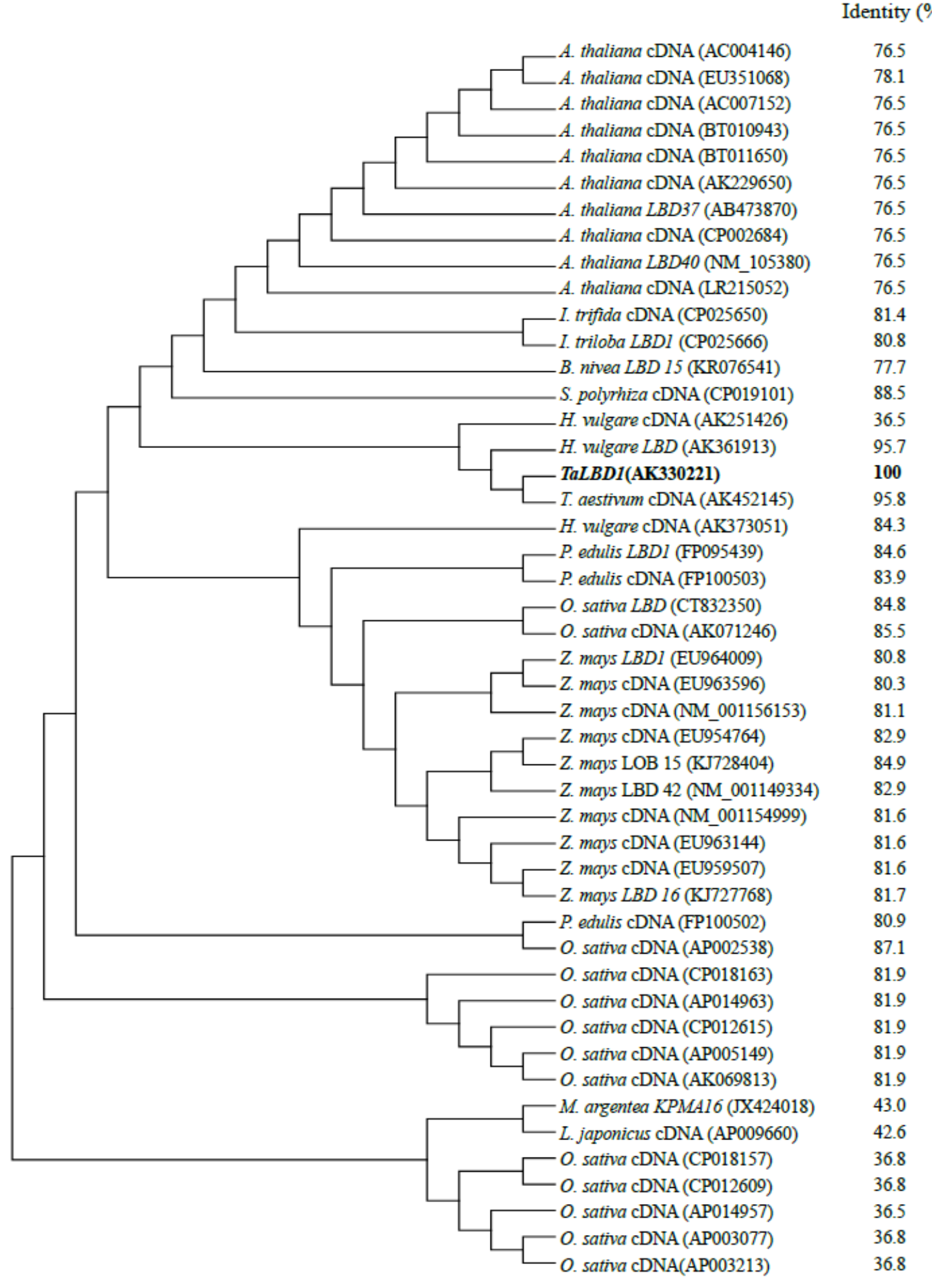

\section{Figure 1}

Phylogenetic relations among TaLBD1 and its homologous genes from various plant species

A. thaliana-Arabidopsis; I. trifida-petunias; I. triloba-sweet potato; B. nivea-ramie; S. polyrhiza-duckweed; $H$. vulgare-barley; T. aestivum-wheat; $P$. edulis-kudzu; $O$. sativa-rice; $Z$. mays-maize; $M$. argentea- marcus silver; L. japonicus- lotus japonicus. 
$\mathbf{A}$

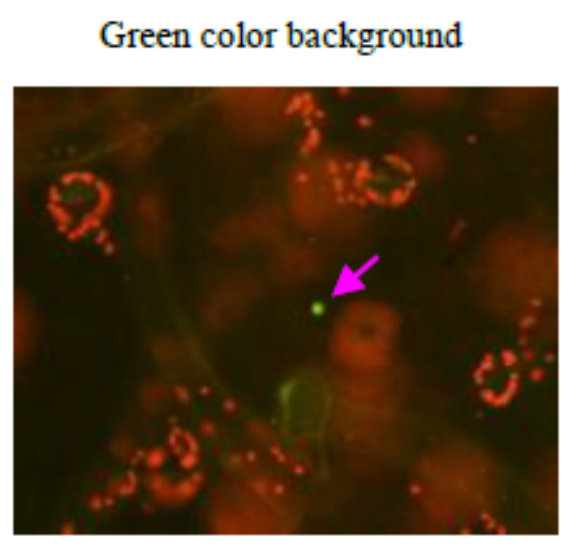

Black and white

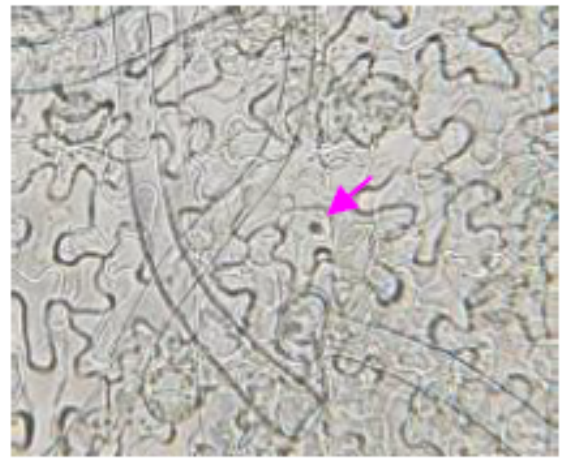

B

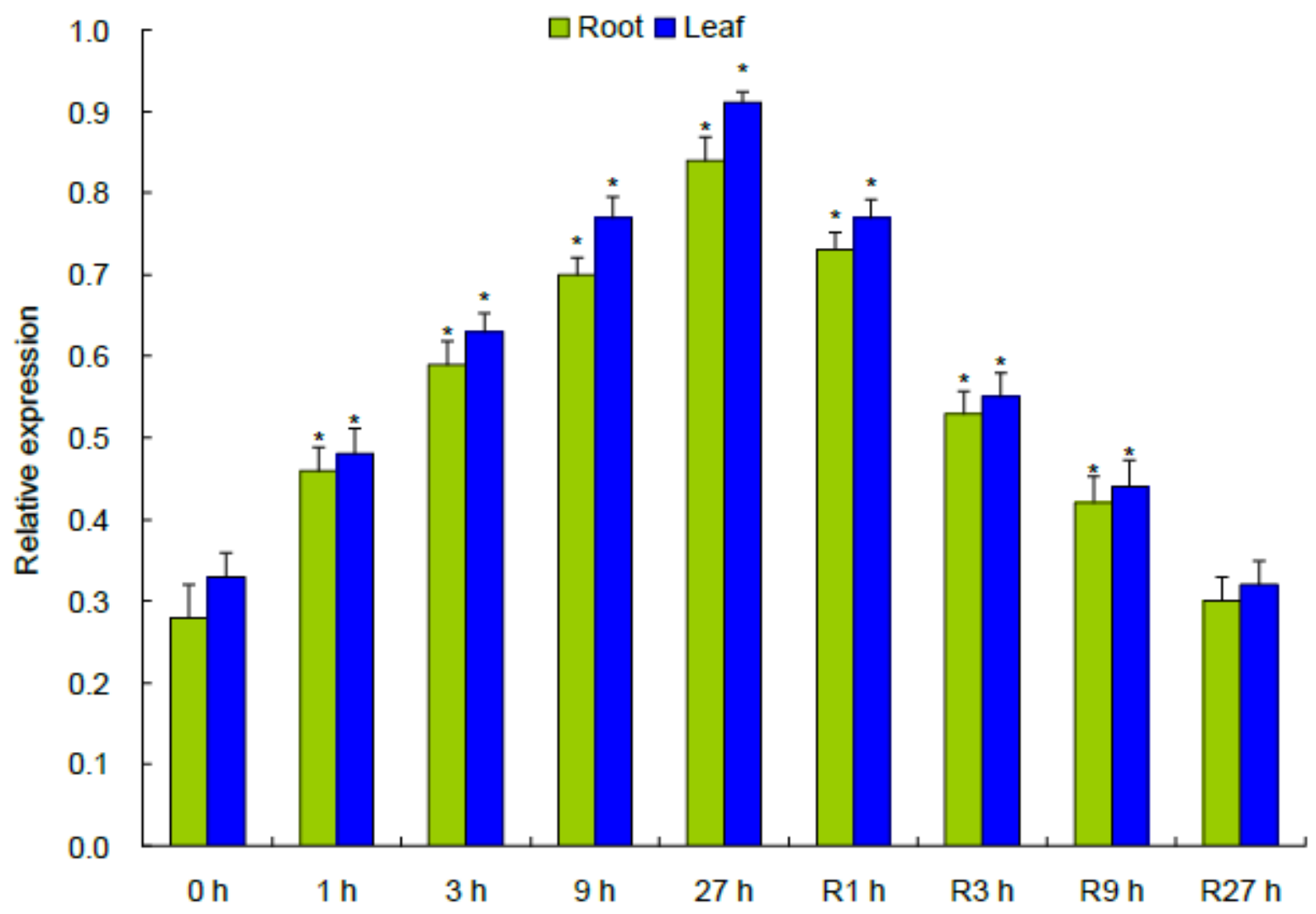

Figure 2

Subcellular localization of TaLBD1 and the expression patterns of TaLBD1 following the external $\mathrm{N}$ treatments

A, The TaLBD1-GFP fusion detected under green microscope. B, expression patterns of TaLBD1 upon the $\mathrm{N}$ starvation treatment. In $\mathbf{A}$, the arrows point to cell nucleus. In $\mathbf{B}, 0 \mathrm{~h}$, time point prior to $\mathrm{N}$ starvation 
stress. $1 \mathrm{~h}, 3 \mathrm{~h}, 9 \mathrm{~h}$, and $27 \mathrm{~h}$, time points after $\mathrm{N}$ starvation treatment. R3 h, R9 h, and R27 h, time points after $\mathrm{N}$ normal recovery treatment. Error bars represent standard errors and symbol * indicates significant differences between transgenic lines and WT calculated by one-way ANOVA with significance level of 0.05 .

$\mathbf{A}$

WT

Line 2

Line 3
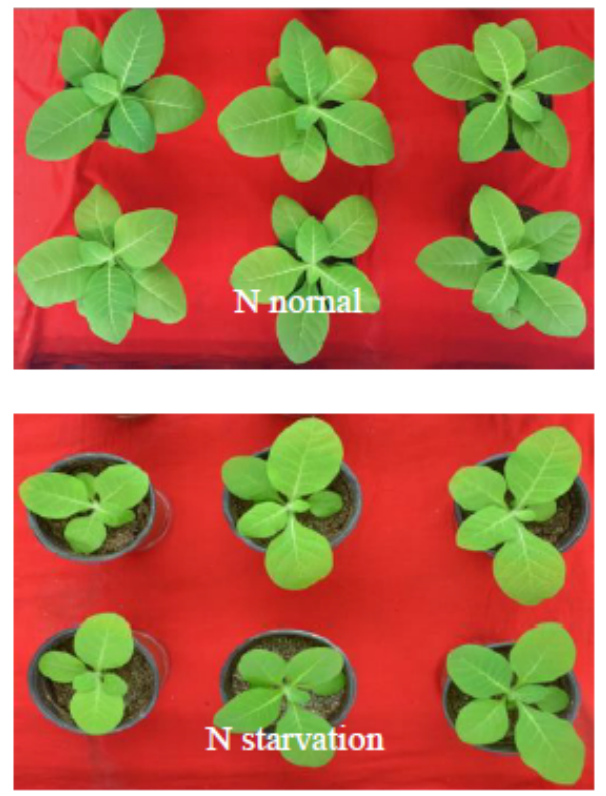

D

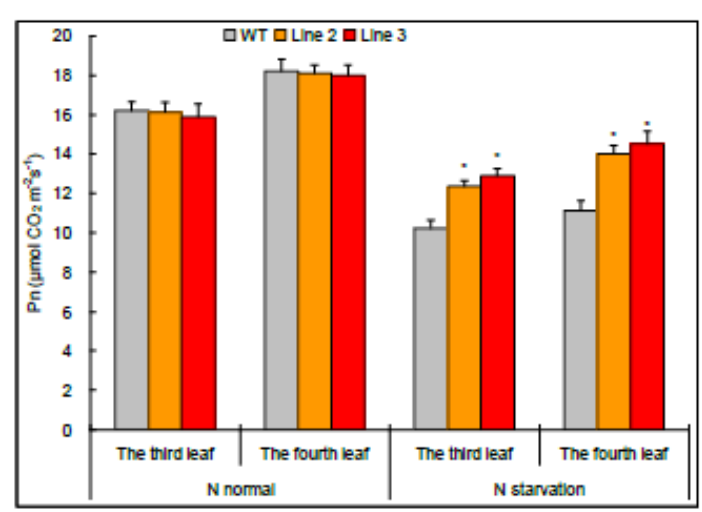

$\mathbf{F}$

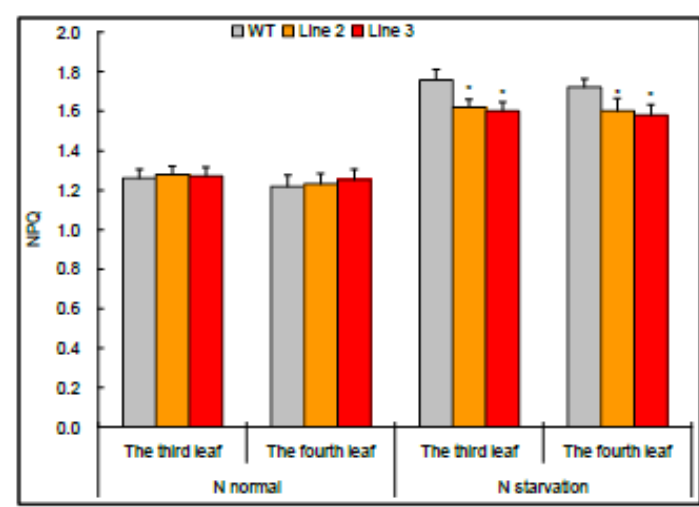

B

WT

Line 2

Line 3

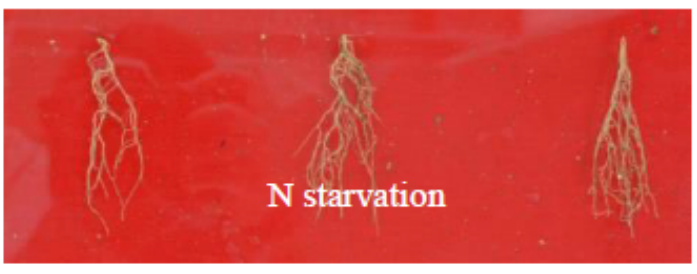

$\mathbf{C}$

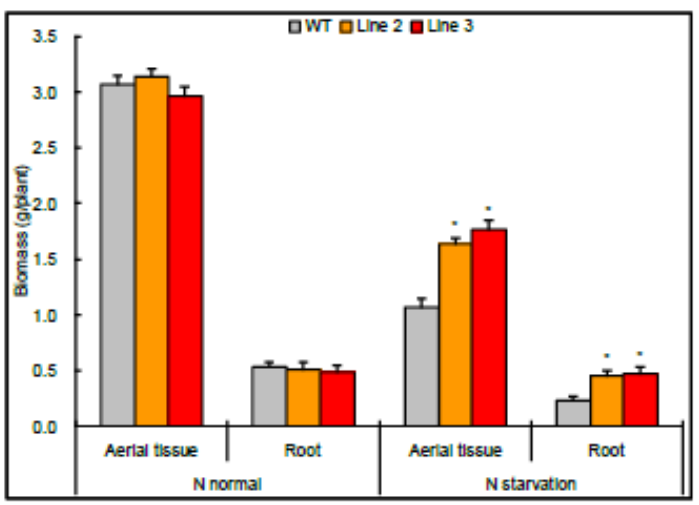

$\mathbf{E}$

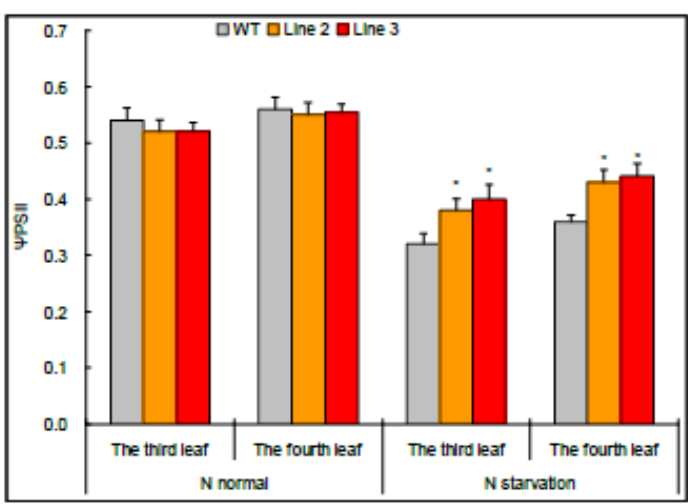




\section{Figure 3}

Phenotypes, biomass, and photosynthetic parameters in TaLBD1 transgenic lines under $\mathrm{N}$ starvation treatment

A, phenotypes of plants. B, phenotypes of roots under $\mathrm{N}$ starvation. C, biomass in aerial tissues and roots. D, photosynthetic rate (Pn). E, photosystem II efficiency (UPSII), F, nonphotochemical quenching (NPQ). WT, wild type. Line 2 and Line 3, two lines with TaLBD1 overexpression. In $\mathbf{C}$ to $\mathbf{F}$, the average values are derived from the triplicate results. Error bars represent standard errors and symbol * indicates significant differences between transgenic lines and WT under same $N$ treatment calculated by one-way ANOVA with significance level of 0.05 . 


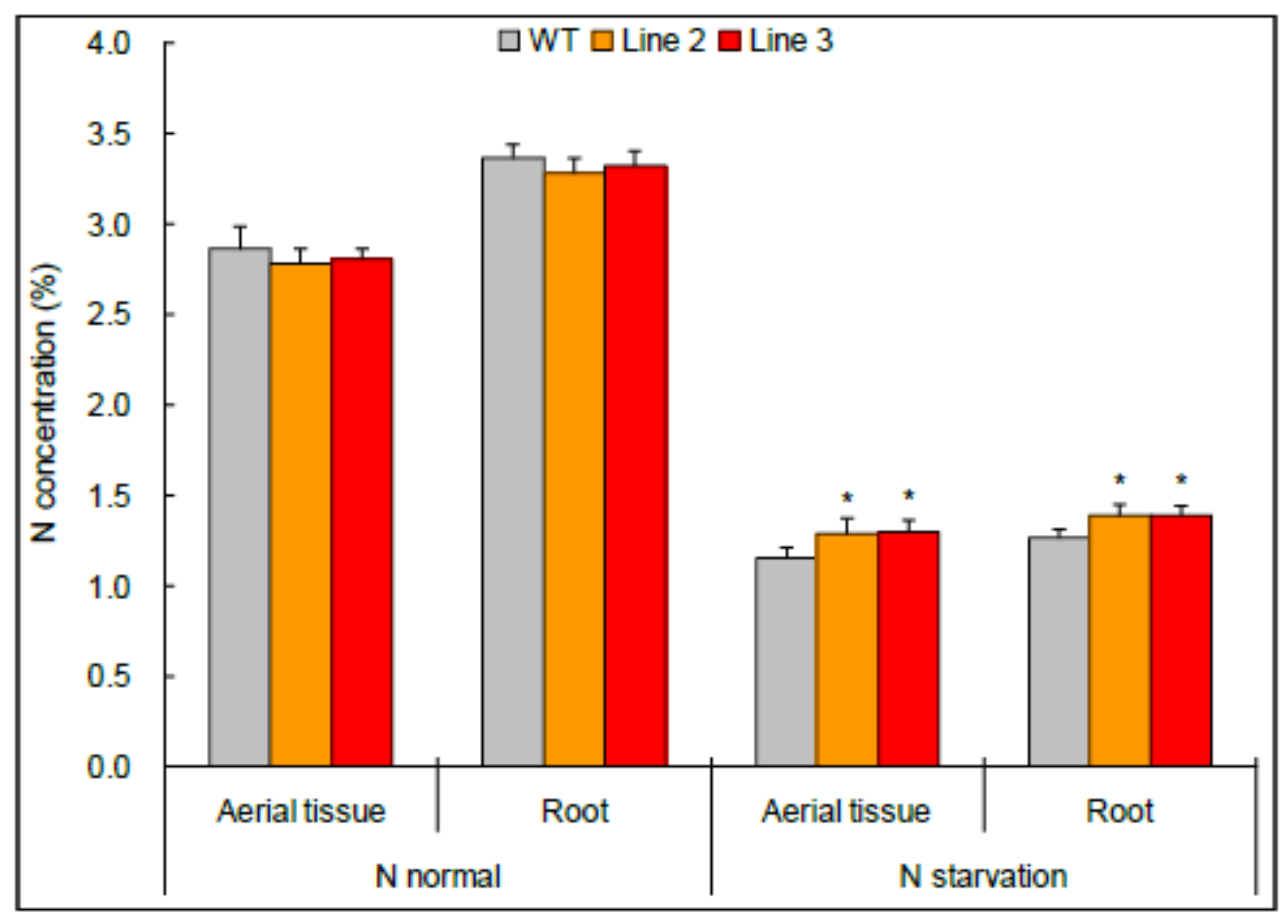

B

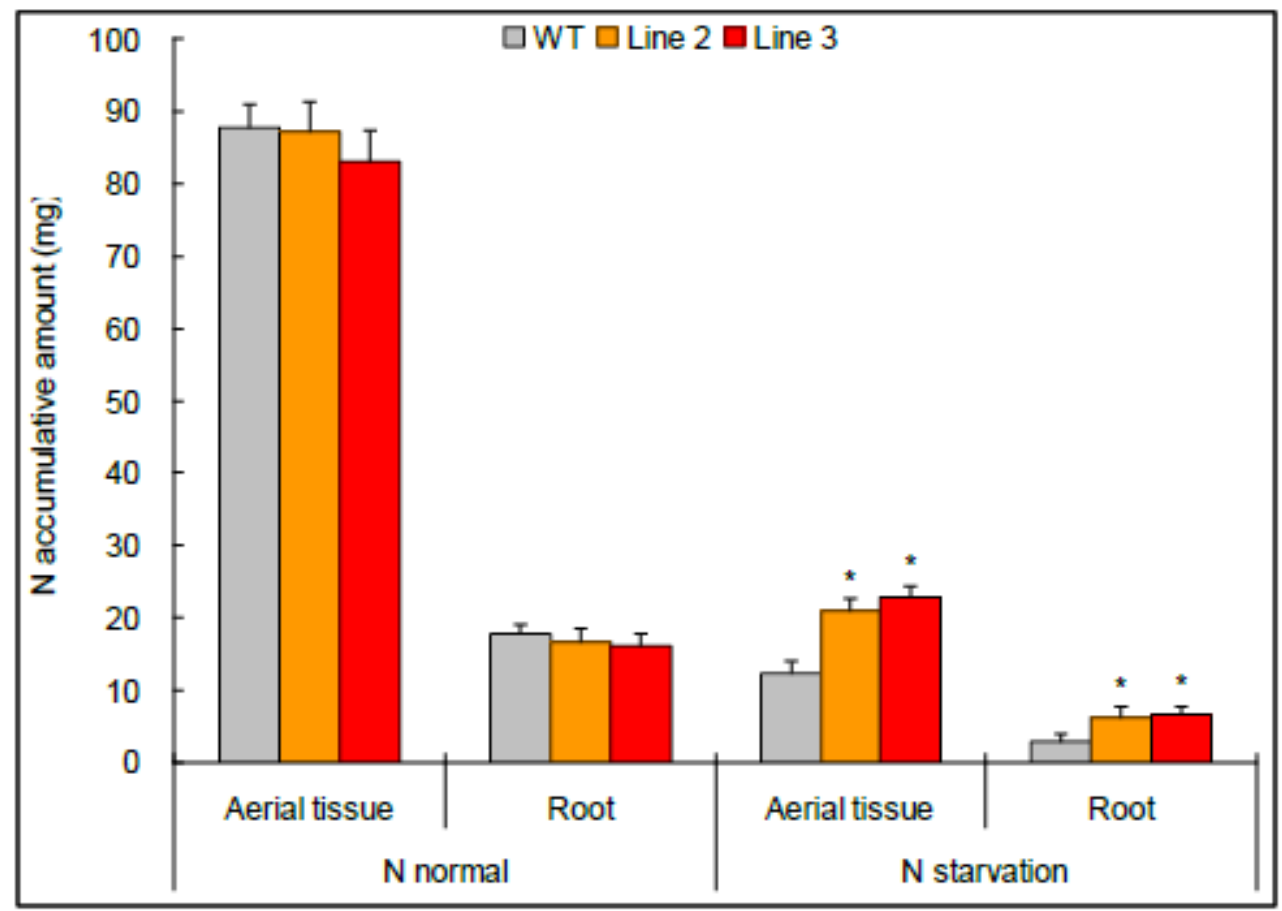

\section{Figure 4}

The $\mathrm{N}$-associated traits in $T a L B D 1$ transgenic lines under the $\mathrm{N}$ starvation treatment

A, $\mathrm{N}$ concentrations in aerial tissues and roots. B, $\mathrm{N}$ accumulative amounts in aerial tissues and roots. WT, wild type. Line 2 and Line 3, lines with TaLBD1 overexpression. The average values are derived from the triplicate results. Error bars represent standard errors and symbol * indicates significant differences 
between transgenic lines and wild type under same $\mathrm{N}$ treatment calculated by one-way ANOVA with significance level of 0.05 .

A

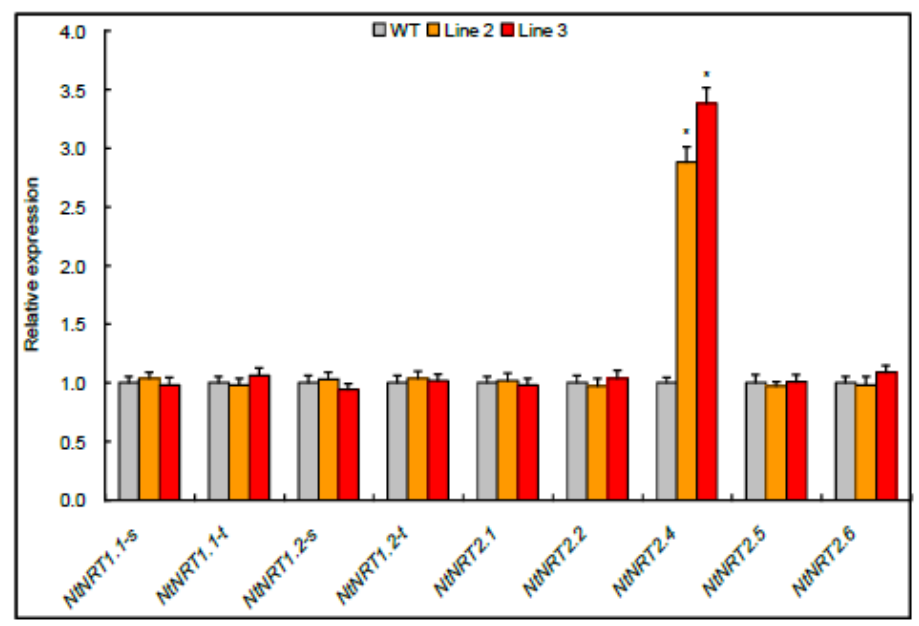

B
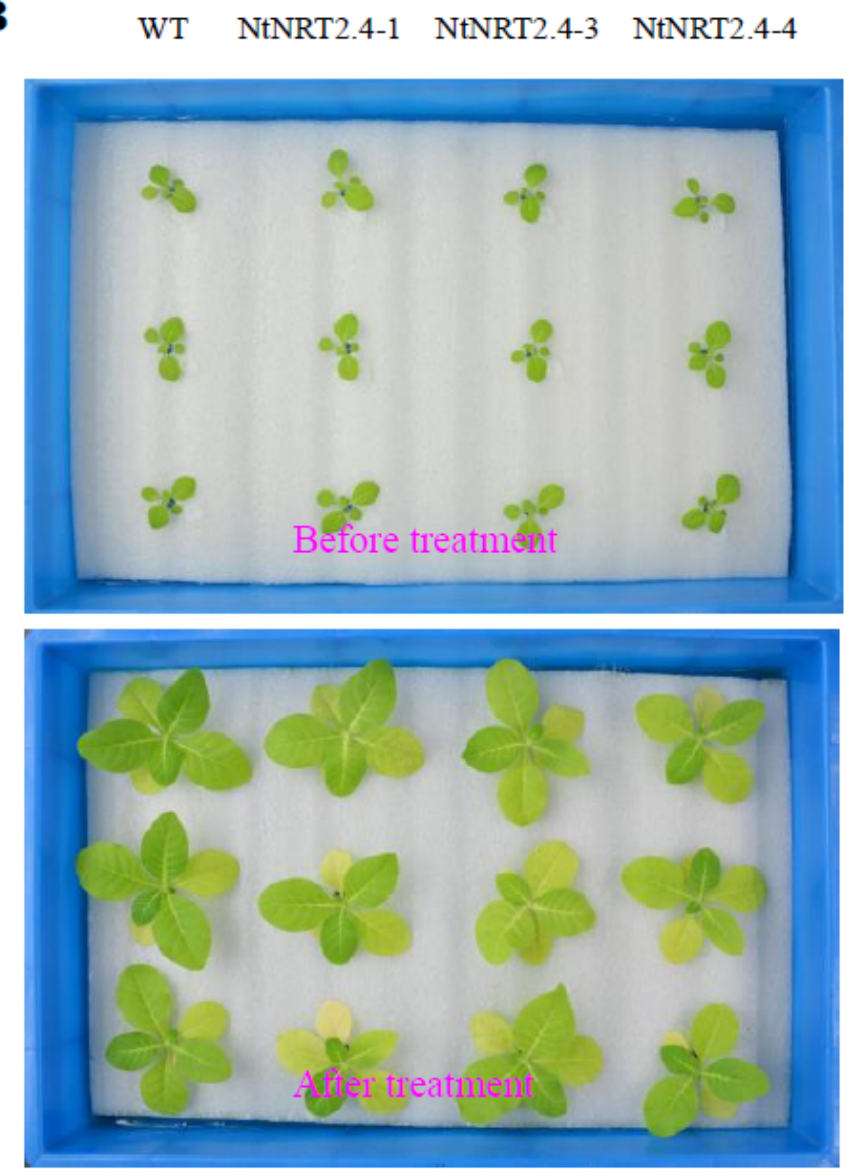

C

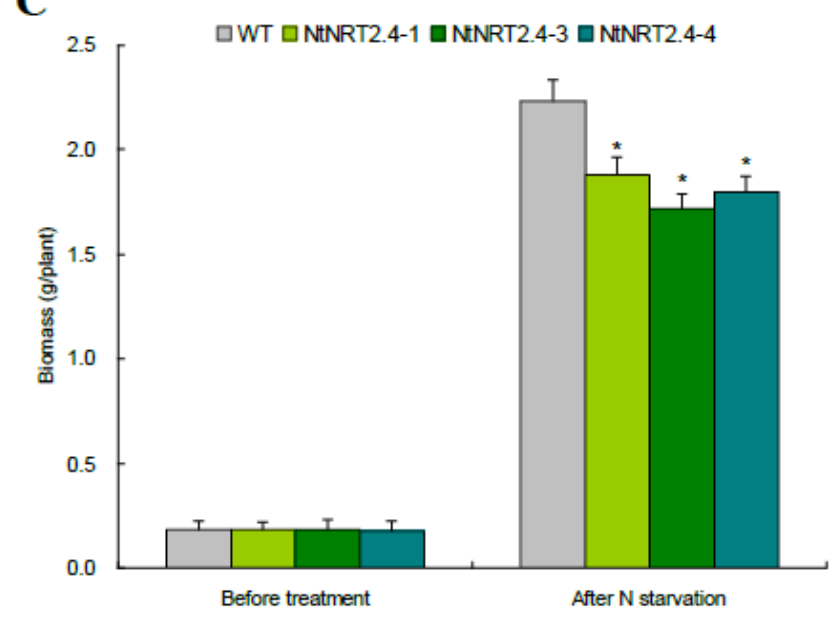

D

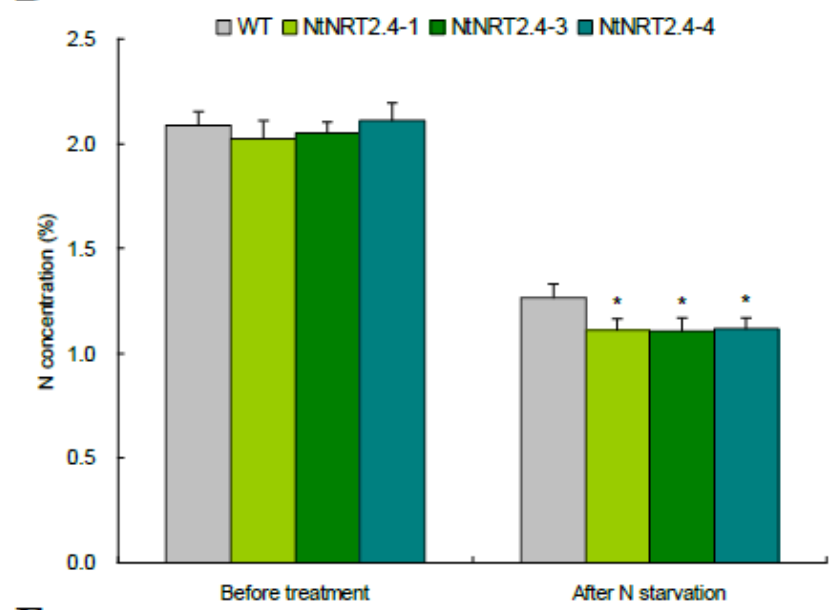

$\mathbf{E}$

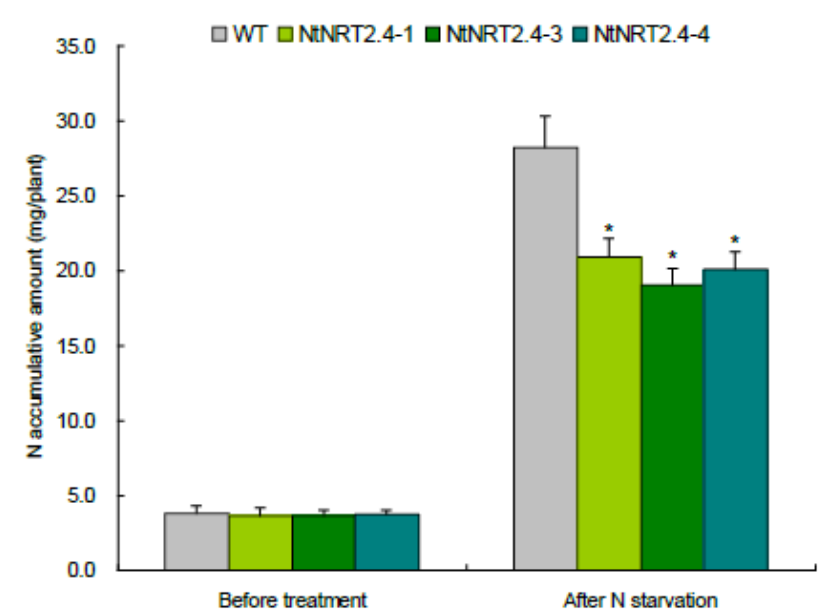

Figure 5

Expression patterns of the NRT family genes and functional analysis on distinct differential NRT gene under $\mathrm{N}$ starvation treatment 
A, Expression patterns of the NRT family genes. B, phenotypes on transgenic lines with NtNRT2.4 knockdown. C, biomass on lines with NtNRT2.4 knockdown. D, N concentrations on lines with NtNRT2.4 knockdown. E, N accumulative amounts on lines with NtNRT2.4 knockdown. In $\mathbf{A}$ and $\mathbf{C}$ to $\mathbf{E}$, the average values are derived from the triplicate results. WT, wild type. NtNRT2.4-1, NtNRt2.4-3 and NtNRT2.4-4, three lines with NtNRT2.4 knockdown. Error bars represent standard errors and symbol * indicates significant differences between transgenic lines and WT calculated by one-way ANOVA with significance level of 0.05 .

A

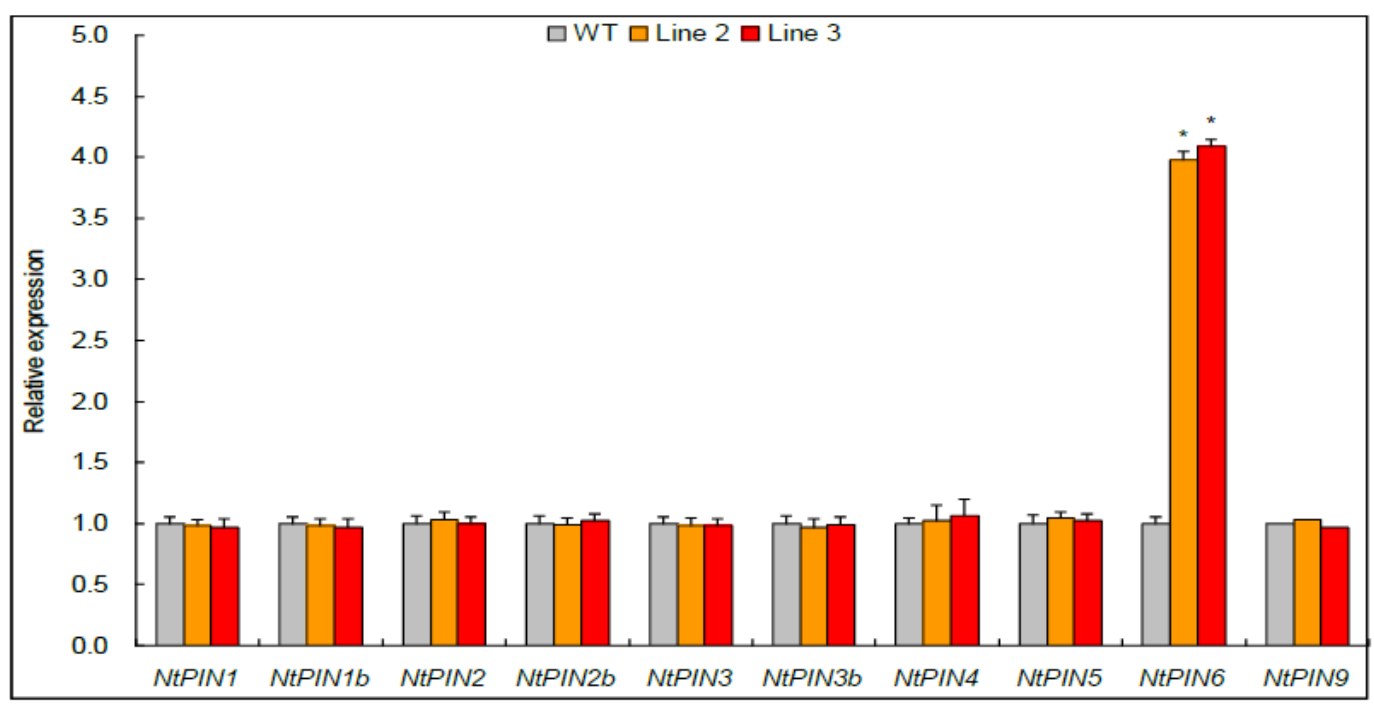

B

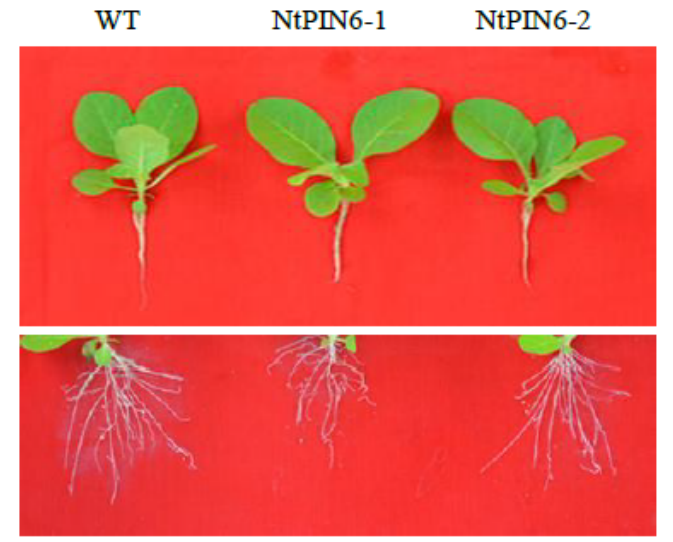

D

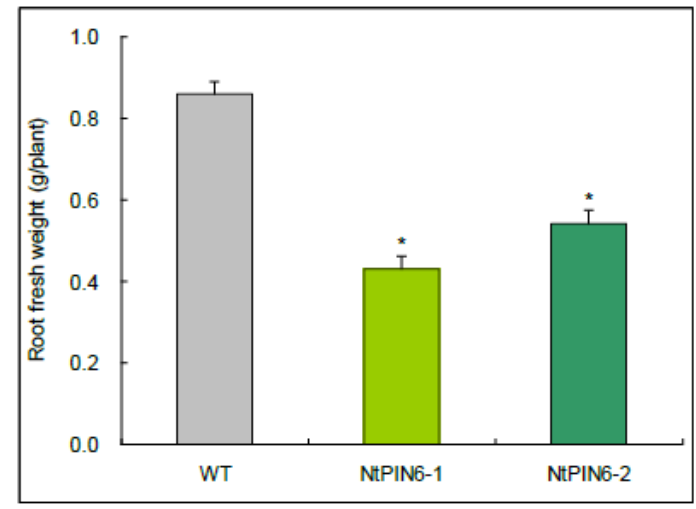

C

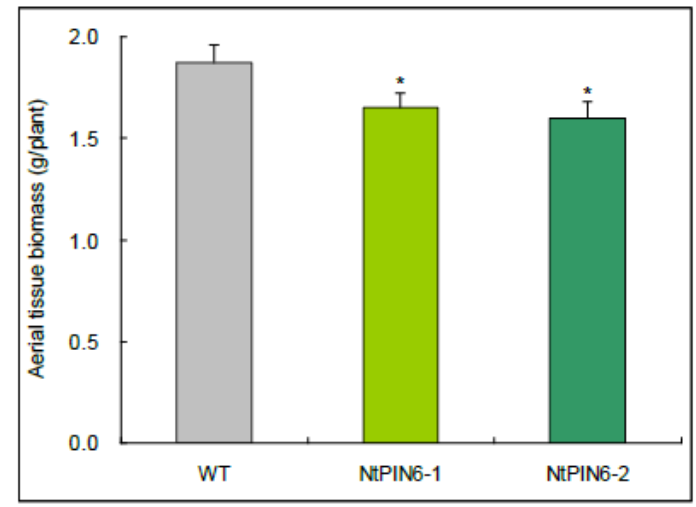

$\mathbf{E}$

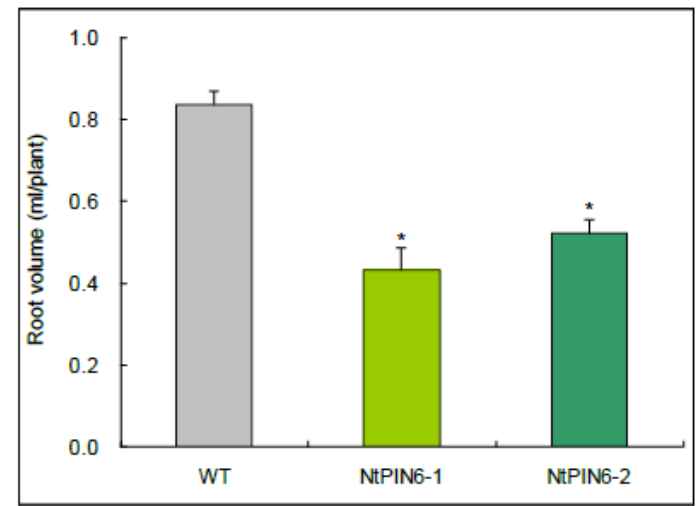




\section{Figure 6}

Expression patterns of the PIN-FORMED family genes and functional analysis on distinct differential PIN gene under $\mathrm{N}$ starvation treatment

A, expression patterns of the PIN-FORMED family genes. B, plant and root phenotypes on transgenic lines with NtPIN6 knockdown. C, biomass on lines with NtPIN6 knockdown. D, Root fresh weights on lines with NtPIN6 knockdown. $\mathbf{E}$, root volumes on lines with NtPIN6 knockdown. In $\mathbf{A}$ and $\mathbf{C}$ to $\mathbf{E}$, the average values are derived from the triplicate results. WT, wild type. NtPIN6-1 and NtPIN6-2, two lines with NtPIN6 knockdown. Error bars represent standard errors and symbol * indicates significant differences between transgenic lines and WT calculated by one-way ANOVA with significance level of 0.05 . 
A

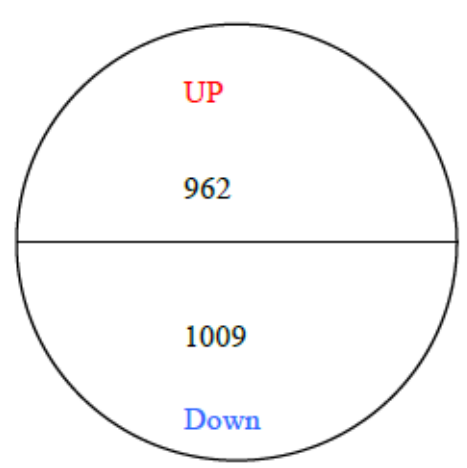

B

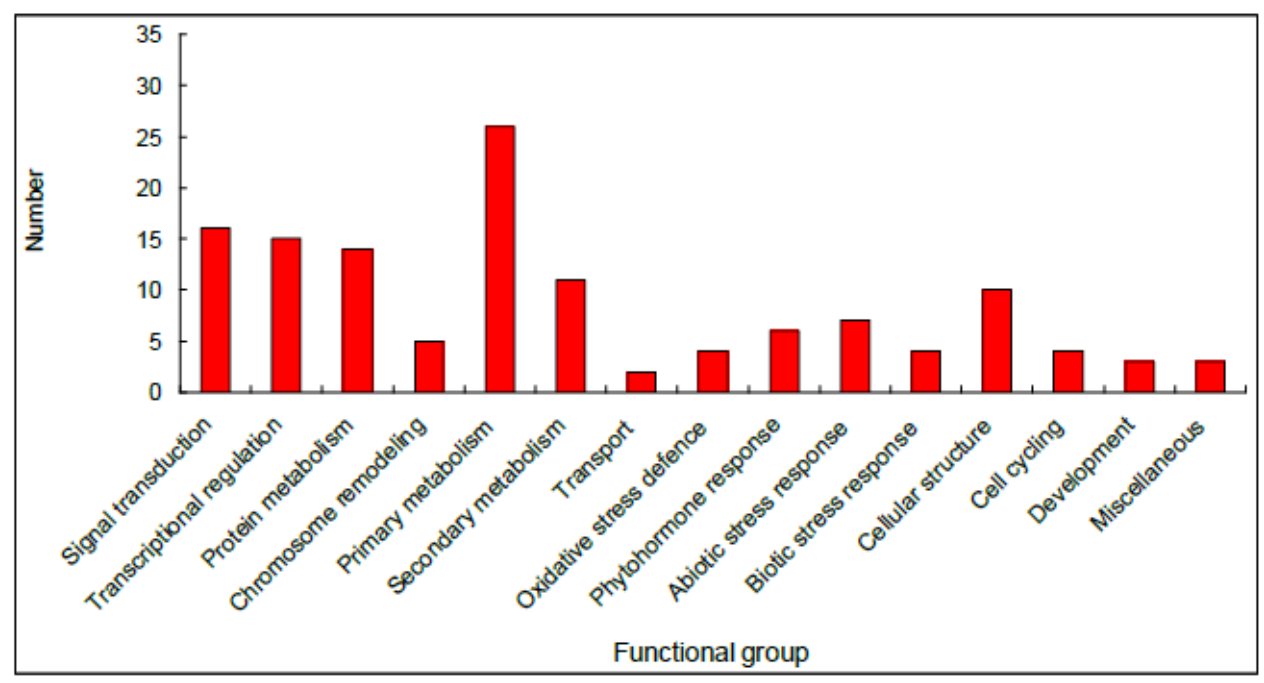

C

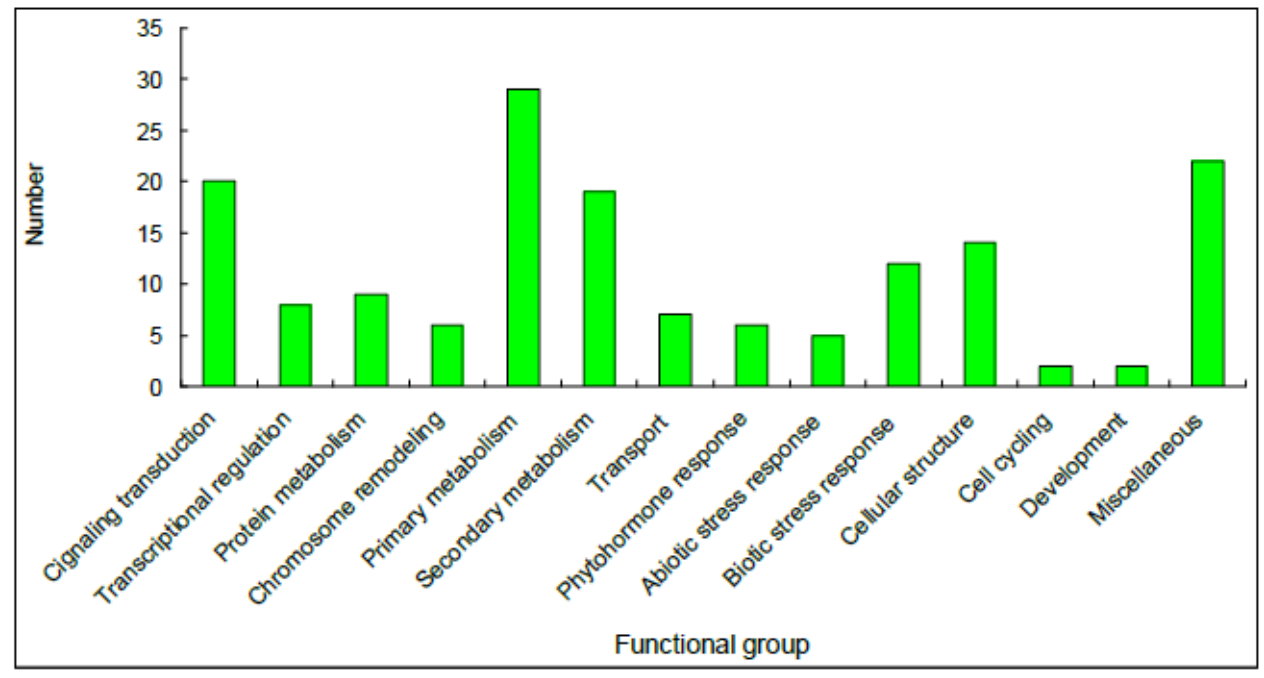

\section{Figure 7}

Functional groups of the DEGs with upregulated and downregulated expression patterns in lines with TaLBD1 overexpression treated by $\mathrm{N}$ starvation stress

A, Venn diagram showing the numbers of the DEGS with upregulated and downregulated expression patterns. B, Functional groups of the upregulated DEGs. C, Functional groups of the downregulated DEGs. 

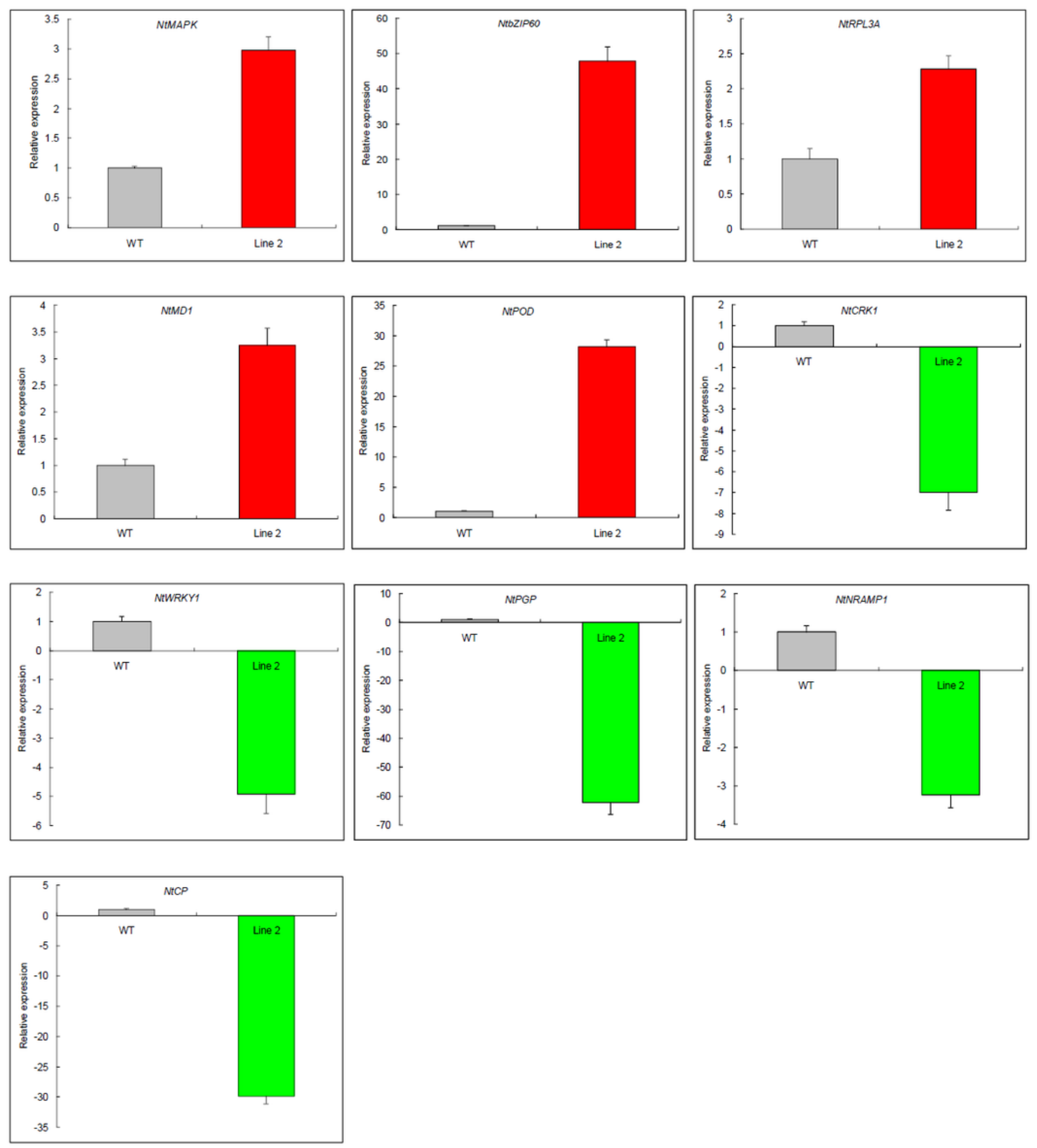

\section{Figure 8}

Expression patterns of ten randomly selected DEGs identified in lines with TaLBD1 overexpression after $\mathrm{N}$ starvation treatment

NtMAPKK (Genbank accession No. BP530009), NtbZIP60 (AB281271), NtRPL3A (AY395738), NtMD1 (AJ299256), and NtPOD (AB044153), five DEGs with upregulated expression pattern in transcriptome 
analysis. NtCRK1 (AF302082), NtWRKY1 (AB022693), NtPGP(X70651), NtNRAMP1 (AB505625), and NtCP (S44869), five DEGs with upregulated expression pattern in transcriptome analysis. Data are normalized by internal standard gene Nttubulin and shown by average plus standard error.

\section{Supplementary Files}

This is a list of supplementary files associated with this preprint. Click to download.

- Supplementaldata.doc 